\title{
Antibiotic Resistance of Strong Biofilm Forming Bacteria Isolated from Different Oral Samples of Patients with Dental Problems
}

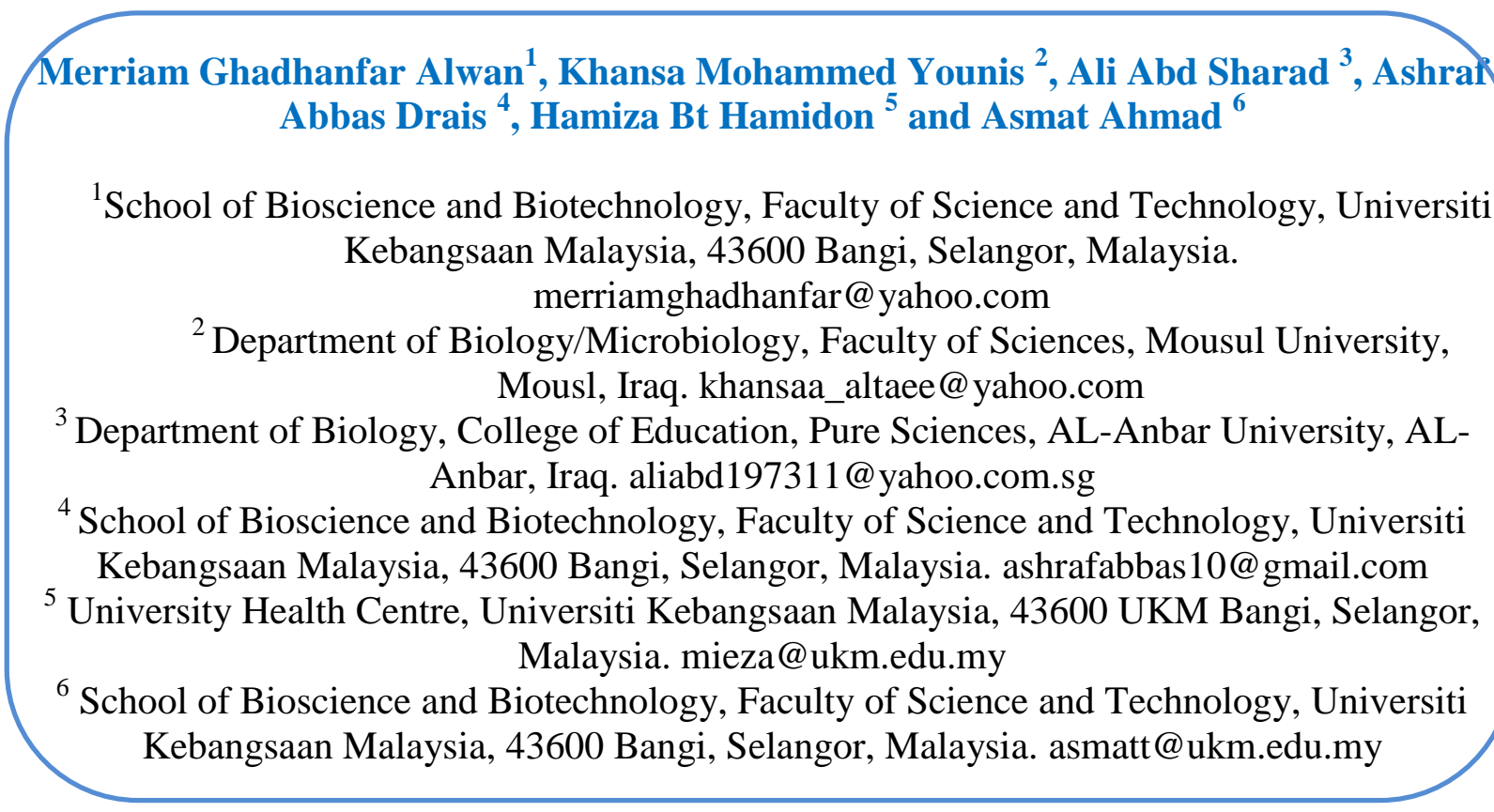

\section{ABSTRACT}

Globally, the wide use of antibiotics in human treatments, animal food supplements, and agriculture practice was the main cause of the emergence of multi antibiotics resistant strains of bacteria which led to a critical health problem. The purpose of this study was to reviewing the antibiotics resistance ability of strong biofilm former isolates isolated from saliva, teeth and necrotic roots canals. Biofilm formation assay was done by using Microtiter plate method (MTP), among 267 bacterial isolates (137 from saliva, 88 from teeth and 42 from necrotic roots canal), 104 isolates (55 from saliva, 32 from teeth and 17 from necrotic roots canals) were considered as strong biofilm former and were screened according to its antibiotics resistance ability. The results showed that almost all the isolates can form visible growing biofilms when compared to the controls, though with difference in the degree of adhesions (strong, moderate and weak). Enterococcus sp. isolated from saliva, teeth and necrotic roots canals was the strongest biofilm former bacteria and as well as the most antibiotic resistant isolates. Further, the study revealed that antimicrobial resistance of bacteria isolated from saliva, teeth and necrotic roots canals of patients with dental caries conditions is associated with biofilm formation.

Keywords: Biofilm formation, Antibiotic resistance, Enterococcus, Saliva, Teeth, Necrotic roots canals

Corresponding Author: Merriam Ghadhanfar Alwan (merriamghadhantar@yahoo.com)

\section{INTRODUCTION}

The oral microbiota has been reported to serve as a reservoir for numerous antimicrobial resistance genes such as those encoding resistance to commonly utilized classes of antimicrobials such as tetracyclines, beta-lactams, and macrolides, which have been extensively used in treating oral infectious conditions, including those of endodontic origin 
[1]. During the conventional resistance against antimicrobial agents by planktonic bacteria, it normally involves the inactivation of the antimicrobial agent, alteration of the targets, and exclusion of the antimicrobial agent [2]. These typical actions involve the acquisition of distinct genetic factors, like genes for $\beta$-lactamase or efflux pumps. One of the most vital characteristics of bacterial biofilm formation is the increased rate of resistance by the constituent microbes to antimicrobial agents as well as other stressors. The nature of the structural of biofilms and the characteristics of the sessile cells, generate resistance towards the antimicrobials, giving rise to an environment that is protected against adverse conditions including the host s defenses [3]. Biofilms may serve as physical diffusion barriers that are capable of preventing antimicrobial agents from getting to their targets. Antimicrobial agents were shown to be capable of penetrating these structures through a thick mixture of exopolysaccharide, DNA, and protein in order to get to their targets [4]. Nevertheless, this mixture was unable to achieve an effective concentration in some parts as a result of the physical and/or chemical properties of the matrix, thereby giving rise to an apparent increase in the rate of resistance. Certainly, recent mathematical modeling has suggested that while limited antimicrobial diffusion may give rise to the death of the outer layer of bacteria, it also stimulates subpopulations of bacteria buried deeper within the biofilm to enact adaptive changes, thereby countering the effect of the antimicrobials [5]. However, limited antimicrobial agent diffusion seems not to be a universal trait shared by all biofilms. Hence there is conflicting data about whether the biofilm matrix is a major contributing factor influencing biofilm resistance [2]. A reduction in penetration and diffusion of the antimicrobial agent via the biofilm matrix has been shown to affect biofilm survival in some instances. For instance, at sub-MIC concentrations of $\beta$-lactam antimicrobials, increased alginate production in Pseudomonas aeruginosa biofilms was induced, and the biofilm matrix of some slime-producing coagulase-negative staphylococci was enhanced. Moreover, even though it was originally suggested that tolerance of biofilms to aminoglycoside was due to transport limitation owing to the binding of these positively charged antimicrobial agents to the negatively charged exopolysaccharide matrix, repeated dosing of antimicrobial agent during treatment may possibly result in saturation of the binding sites $[6,7,8,9]$. This study aimed to perform phenotypic screening of the strong biofilm former bacterial isolates from saliva, teeth, and necrotic root canals according to their multi-antibiotic resistance ability.

\section{MATERIALS AND METHODS}

\section{Bacterial isolates}

This study was carried out in the department of Microbiology, school of bioscience and biotechnology, faculty of science and technology, National University Malaysia. All bacterial isolates isolated from saliva, teeth and necrotic roots canals samples by employing standard bacteriologic techniques. Preliminary tests were done to classify the isolates according to the morphological and cultural characteristics. For further identification, biochemical tests were used. As a result, 267 isolates (137 from saliva, 88 from teeth and 42 from necrotic roots canals) were identified to the genus level.

\section{Biofilm formation assay by using Microtiter plate method (MTP)}

This technique of quantitative detection of biofilm using 96 well microtitre plate employing the ability of the isolates to form a biofilm on abiotic surfaces was used. This method was used as mentioned by [10]. All tests were carried out in triplicate, and the strains were divided into the following categories: no biofilm producer, weak biofilm producer, moderate biofilm producer, or strong biofilm producer [11]. For this, it was necessary to establish the cutoff value (ODc). The ODc was defined as three standard deviations (SD) above the mean 
OD of the negative control (uninoculated medium): ODc $=$ average OD of negative control + ( $3 \times \mathrm{SD}$ of negative control). Based on the OD values:

$\mathrm{OD} \leq \mathrm{ODc}=$ non biofilm producers;

$\mathrm{ODc}<\mathrm{OD} \leq 2 \times \mathrm{ODc}=$ weak biofilm producer;

$2 \times \mathrm{ODc}<\mathrm{OD} \leq 4 \times \mathrm{ODc}=$ moderate biofilm producers;

$3 \times \mathrm{ODc}<\mathrm{OD}=$ strong biofilm producers

\section{Antibiotics Susceptibility Assay}

Antibiotic resistance of the strong biofilm forming isolates (saliva $n=55$, teeth $n=32$ and necrotic roots canals $n=17$ ) were determined on Muller \& Hinton Agar (Oxoid) using KirbyBauer disk diffusion method [12]. The antibiotics selected for the study comprised of: kanamycin $\mathrm{K}(30 \mu \mathrm{g})$, vancomycin VA $(30 \mu \mathrm{g})$, amoxycillin AML $(10 \mu \mathrm{g})$, erythromycin E $(30 \mu \mathrm{g})$, ampicillin AMP $(10 \mu \mathrm{g})$, cephalothin KF $(30 \mu \mathrm{g})$, chloramphenicol C $(30 \mu \mathrm{g})$, tetracycline TE $(30 \mu \mathrm{g})$, ciprofloxacin CIP $(10 \mu \mathrm{g})$, nalidixic acid NA $(30 \mu \mathrm{g})$, gentamicin CN $(10 \mu \mathrm{g})$, novobiocin NV $(30 \mu \mathrm{g})$, carbenicillin CAR $(100 \mu \mathrm{g})$, oxacillin OX $(5 \mu \mathrm{g})$, penicillin $\mathrm{G}$ (10 UI) and streptomycin S $(10 \mu \mathrm{g})$. All the antibiotic discs were procured from Oxoid (UK). E. faecalis ATCC 33186 was used as control strains.

\section{RESULTS AND DISCUSSION}

Table 1 biofilm formation ability among bacterial isolates from saliva

\begin{tabular}{|c|c|c|c|c|}
\hline Genus & $\begin{array}{l}\text { Isolates } \\
\text { number }\end{array}$ & Strong & Moderate & Weak \\
\hline Enterococcus sp. & 20 & 16 & 4 & 0 \\
\hline Streptococcus sp. & 18 & 10 & 6 & 2 \\
\hline Staphylococcus sp. & 16 & 10 & 4 & 2 \\
\hline Bacillus sp. & 9 & 5 & 2 & 2 \\
\hline Actinomyces sp. & 3 & 0 & 2 & 1 \\
\hline Escherichia coli & 11 & 6 & 1 & 4 \\
\hline Salmonella sp. & 4 & 0 & 3 & 1 \\
\hline Shigella sp. & 2 & 0 & 2 & 0 \\
\hline Pseudomonas sp. & 5 & 3 & 2 & 0 \\
\hline Klebsiella sp. & 4 & 0 & 3 & 1 \\
\hline Clostridium sp. & 8 & 0 & 6 & 2 \\
\hline Micrococcs sp. & 4 & 0 & 4 & 0 \\
\hline Enterobacter sp. & 6 & 0 & 5 & 1 \\
\hline Proteus sp. & 7 & 5 & 1 & 1 \\
\hline Peptostreptococcus sp. & 4 & 0 & 2 & 2 \\
\hline Lactobacillus sp. & 3 & 0 & 3 & 0 \\
\hline Fusobacterium sp. & 3 & 0 & 0 & 3 \\
\hline Eubacterium sp. & 2 & 0 & 2 & 0 \\
\hline Provotella sp. & 2 & 0 & 1 & 1 \\
\hline Bacteroides sp. & 4 & 0 & 3 & 1 \\
\hline Aerococcus sp. & 2 & 0 & 1 & 1 \\
\hline Total & 137 & 55 & 57 & 25 \\
\hline
\end{tabular}

\section{Microtiter Plate Method (Crystal Violet Method)}

The findings from this screening technique showed that almost all the isolates can form visible growing biofilms when compared to the controls, though with difference in the degree 
of adhesions. The results showed that $16(80 \%)$ of the Enterococcus sp. isolates from saliva considered as a strong biofilm former while 4 (20\%) showed moderate biofilm formation. For Streptococcus sp., Staphylococcus sp., Bacillus sp., E. coli, Pseudomonas sp., Proteus sp. the numbers of the strong biofilm former isolates were 10 (55.5\%), $10(62.5 \%), 5(55.5 \%), 6$ $(54.5 \%), 3(60 \%)$, and $5(71.4 \%)$ respectively (Table 1$)$.

The results obtained from this method showed that 8 (57.1\%) of the Enterococcus sp. isolates from teeth can be considered as a strong biofilm former, while $4(28.6 \%)$ isolates were considered moderate and $2(14.3 \%)$ isolates were considered as weak biofilm formers. Also the numbers of the strong isolates were 5 (29.4\%), 7 (43.7\%), 1 (33.3\%), $4(44.4 \%), 2$ (28.5\%), 1 (33.3\%), 2 (22.2\%) and 2 (40\%) for Streptococcus sp., Staphylococcus sp., Bacillus sp., E. coli, Pseudomonas sp., Klebsiella sp., Enterobacter sp. and Proteus sp. respectively (Table 2).

Table 2 biofilm formation ability among bacterial isolates from teeth

\begin{tabular}{|c|c|c|c|c|}
\hline Genus & $\begin{array}{l}\text { Isolates } \\
\text { numbers }\end{array}$ & Strong & Moderate & Weak \\
\hline Enterococcus sp. & 14 & 8 & 4 & 2 \\
\hline Streptococcus sp. & 17 & 5 & 9 & 3 \\
\hline Staphylococcus sp. & 16 & 7 & 6 & 3 \\
\hline Bacillus sp. & 3 & 1 & 2 & 0 \\
\hline Actinomyces sp. & 2 & 0 & 2 & 0 \\
\hline E. coli & 9 & 4 & 3 & 2 \\
\hline Pseudomonas sp. & 7 & 2 & 3 & 2 \\
\hline Klebsiella sp. & 3 & 1 & 2 & 0 \\
\hline Enterobacter sp. & 9 & 2 & 6 & 1 \\
\hline Proteus sp. & 5 & 2 & 2 & 1 \\
\hline Lactobacillus sp. & 3 & 0 & 2 & 1 \\
\hline Total & 88 & 32 & 41 & 15 \\
\hline
\end{tabular}

One of the important results that observed during the employment of the MTP method was the high percentage of the strong biofilm formers among the Enterococcus sp. isolates, isolated from necrotic roots canals which the number was $8(88.9 \%)$ followed by Streptococcus sp. 5 (71.4\%), Clostridium sp. 2 (66.6\%), Staphylococcus sp. 1 (25\%) and Bacillus sp. 1 (20\%). (Table 3). In this study, it was noted that the ability of Enterococcus sp. isolates to produce strong biofilm formation was higher than the others, and this was due to many reasons. Enterococci have been reported as important organisms in periodontal infection [13]. The adherence and production of a biofilm by E. faecalis on different biomaterials have been demonstrated, and the capacity of enterococci to bind to various medical devices has been associated with the ability of enterococci to produce biofilms [14]. The number of genetic factors known to be involved in biofilm production has increased in recent years, due to the availability of genomic and proteomic approaches, but much more research is needed to allow a better understanding of the regulation of biofilm production [15]. These genetic factors such as the presence of esp gene (Enterococcal surface protein), gelE gene (Secretory metalloprotease gelatinase E) and bee gene (Biofilm enhancer in 
Enterococcus/a putative cell wall-anchored protein) [15]. A complete understanding of the role of genetic and environmental factors in the development of biofilm may lead to improved strategies for biofilm control among enterococci.

Table 3 biofilm formation ability among bacterial isolates from necrotic roots canals

\begin{tabular}{|l|c|c|c|c|}
\hline \multicolumn{1}{|c|}{ Genus } & $\begin{array}{c}\text { Isolates } \\
\text { number }\end{array}$ & Strong & Moderate & Weak \\
\hline Enterococcus sp. & 9 & 8 & 1 & 0 \\
\hline Streptococcus sp. & 7 & 5 & 2 & 0 \\
\hline Staphylococcus sp. & 4 & 1 & 2 & 1 \\
\hline Bacillus sp. & 5 & 1 & 3 & 1 \\
\hline Actinomyces sp. & 3 & 0 & 1 & 2 \\
\hline Clostridium sp. & 3 & 2 & 1 & 0 \\
\hline Peptostreptococcus sp. & 4 & 0 & 2 & 2 \\
\hline Fusobacterium sp. & 2 & 0 & 2 & 0 \\
\hline Provotella sp. & 1 & 0 & 1 & 0 \\
\hline Bacteroides sp. & 3 & 0 & 3 & 0 \\
\hline Chromobacterium sp. & 1 & 0 & 0 & 1 \\
\hline Total & 42 & 17 & 18 & 7 \\
\hline
\end{tabular}

\section{Antibiotic resistance among saliva isolates}

Fifty-five strong biofilm producing isolates were subjected to screening its ability to resist sixteen type of antibiotics. Figure (1) showed the percentage of antibiotics resistance among the gram-positive strong biofilm former isolates of saliva $(n=41)$. These isolates identified to four genera as Enterococcus sp., Streptococcus sp., Staphylococcus sp. and Bacillus sp. It is apparent from this figure that all the isolates were resistant to the nalidixic acid and penicillin $\mathrm{G}$, also all the isolates resistant to streptomycin except one isolate of Bacillus sp. (S96). The results also indicated that all the Enterococcus sp. isolates were resistance to the all antibiotics except ampicillin and amoxicillin, which two isolates (S88 and S106) were sensitive to ampicillin and five isolates (S52, S88, S98, S106 and S131) were sensitive to amoxicillin (Table 4). The observed increase in antibiotic resistance among Enterococcus sp. isolates could be attributed to the strong biofilm formation ability of these isolates science many studies reported the number of genetic factors known to be involved in biofilm production [15], in addition to that these organisms have developed resistance to virtually all antimicrobials currently used in clinical practice using a diverse number of genetic strategies [16]. Due to this ability to recruit antibiotic resistance determinants, MDR enterococci display a wide repertoire of antibiotic resistance mechanisms including modification of drug targets, inactivation of therapeutic agents, overexpression of efflux pumps and a sophisticated cell envelope adaptive response that promotes survival in the human host and the nosocomial environment [16]. Figure (2) presents an overview of the antibiotics resistance among gramnegative strong biofilm former isolates from saliva $(n=14)$. The results showed that all the isolates have the ability to resist vancomycin, novobiocin, erythromycin, penicillin, oxacillin and cephalothin, while this resistance decreased with other types of antibiotics (ciprofloxacin, gentamycin, carbenicillin and streptomycin). The results indicate that all E. coli isolates $(\mathrm{n}=6)$ were sensitive to the tetracycline, gentamycin, carbenicillin, nalidixic acid and chloramphenicol, while Proteus sp. isolates $(\mathrm{n}=5)$ were sensitive to gentamycin and 
chloramphenicol only. For Pseudomonas sp. isolates $(n=3)$, the results showed that all the isolates (S3, S33 and S97) sensitive only to carbenicillin while the resistance to the other types of antibiotics were varied. Resistance rates are increasing among several problematic gram-negative pathogens that are often responsible for serious nosocomial infections [17].

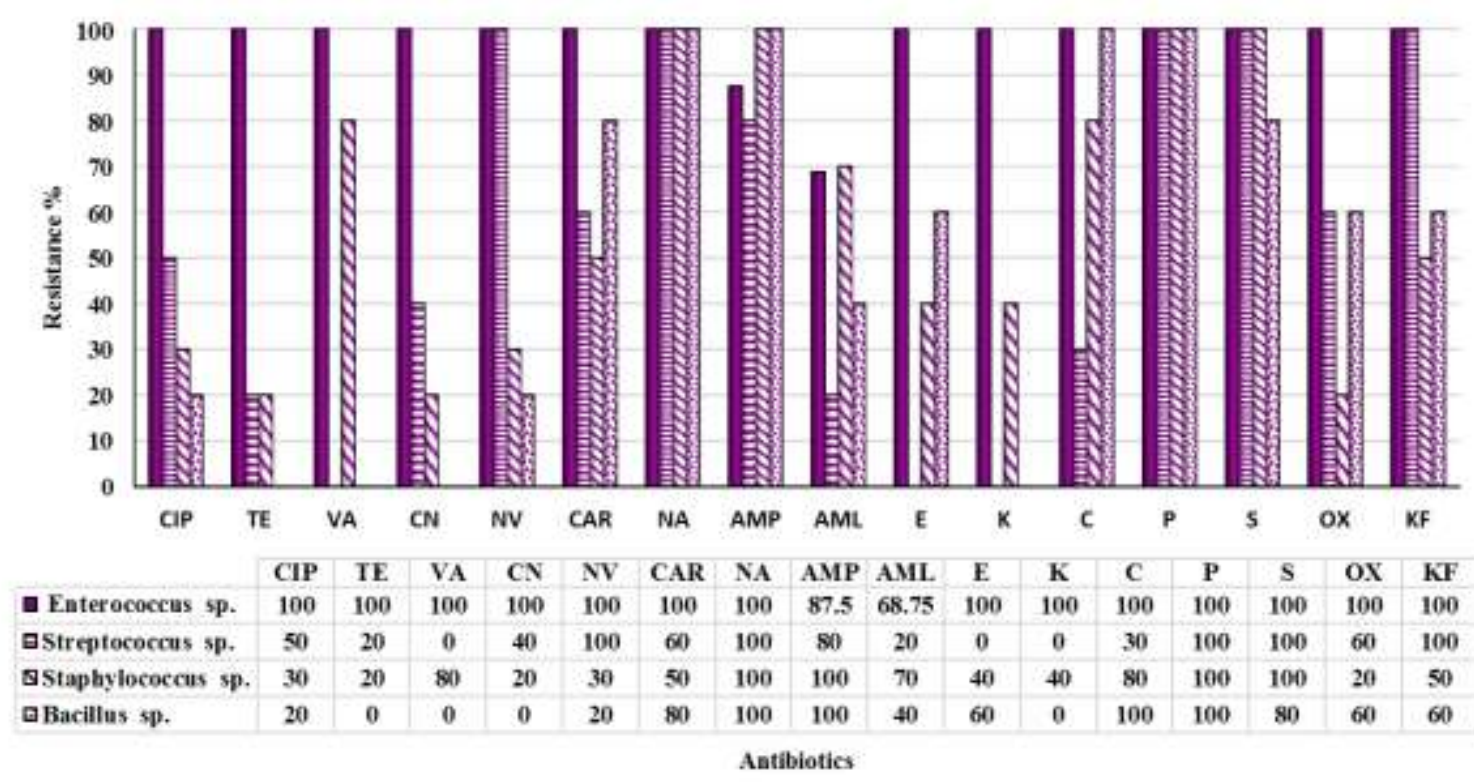

- Enterococcus sp. EStreptococcus sp. DStaphylococcus sp. $\mathrm{B}$ Bacillus sp.

Fig 1 Antibiotics resistance of gram-positive isolates of saliva samples

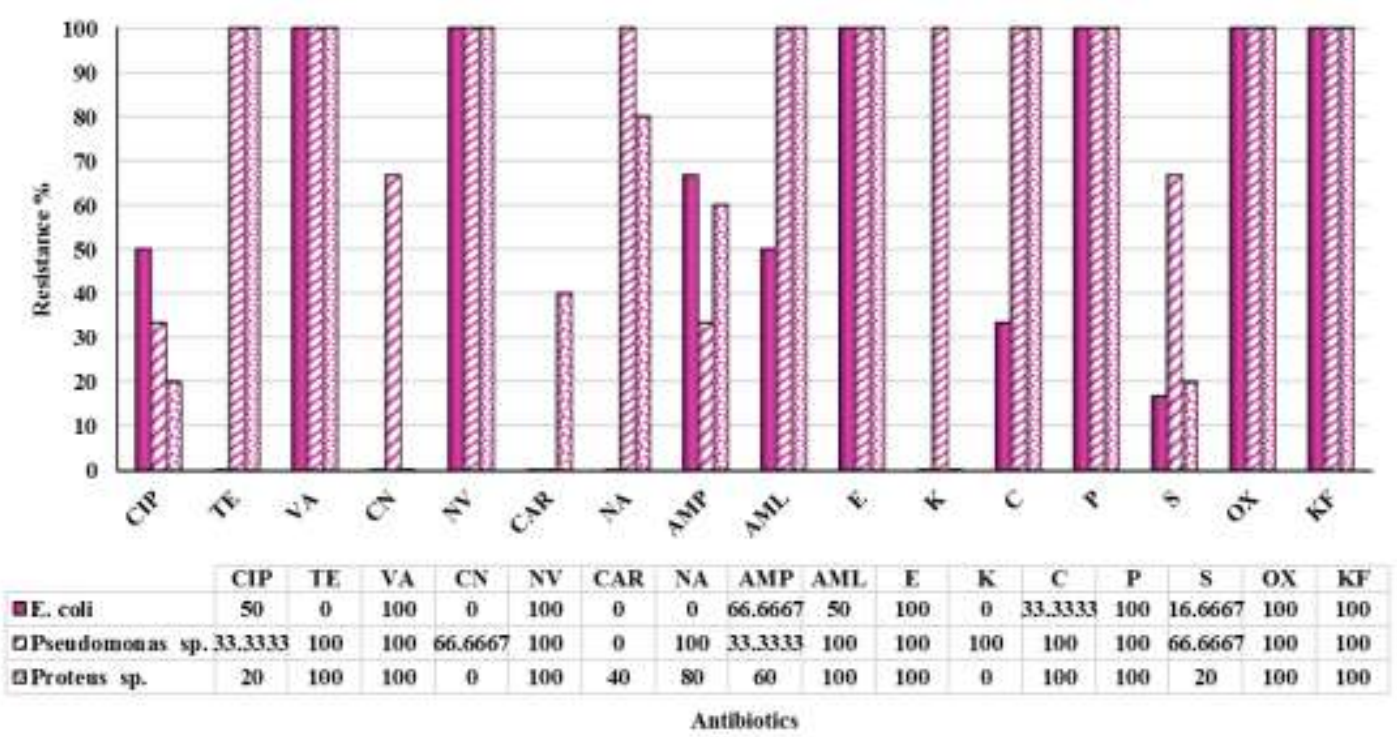

घE. coli $\square$ Pseudomonas sp. uProleus sp.

Fig 2 Antibiotics resistance of gram-negative isolates of saliva samples 
Table (4) Antibiogram pattern of the strong biofilm former isolates from saliva

\begin{tabular}{|c|c|}
\hline Isolates codes & Antibiogram \\
\hline $\begin{array}{lll}\text { S1, S2, } & \text { S16, S22, } & \text { S11, S40, S46, } \\
\text { S98, S124, } & \text { S131, } & \text { S133 } \\
\text { (Enterococcus } \text { sp.) } & & \end{array}$ & CIP,TE,VA,CN,NV,CAR,NA,AMP,AML,E,K,C,P,S,OX,KF \\
\hline S3, S33 (Pseudomonas sp.) & TE,VA,CN,NV,NA,AMP,AML,E,K,C,P,S,OX,KF \\
\hline S4, S41(Escherichia coli) & CIP,VA,NV,E,P,OX,KF \\
\hline S12 (Streptococcus sp.) & CN,NV,CAR,NA,AMP,AML,P,S,KF \\
\hline S14 (Proteus sp.) & CIP,TE,VA,NV,AMP,AML,E,C,P,S,OX,KF \\
\hline S18 (Staphylococcus sp.) & CIP,TE,VA,NA,AMP,AML,K,P,S,KF \\
\hline S30, S43 (Proteus sp.) & TE,VA,NV,NA,AMP,AML,E,C,P,OX,KF \\
\hline S34 (Staphylococcus sp.) & CIP,TE,VA,CN,NA,AMP,AML,P,S,KF \\
\hline S36 (Bacillus sp.) & CIP,TE,VA,CN,NV,NA,AMP,AML,E,C,P,S,OX,KF \\
\hline S44 (Bacillus sp.) & CAR,NA,AMP,E,C,P,OX,KF \\
\hline S49 (Staphylococcus sp.) & CIP,VA,CN,NA,AMP,AML,K,C,P,S,KF \\
\hline S52, S56 (Enterococcus sp.) & CIP,TE,VA,CN,NV,CAR,NA,E,K,C,P,S,OX,KF \\
\hline S55, S86 (Proteus sp.) & TE,VA,NV,CAR,NA,AML,E,C,P,OX,KF \\
\hline S58 S91 (Staphylococcus sp.) & VA,NA,AMP,AML,C,P,S \\
\hline S64 (Streptococcus sp.) & CN,NV,CAR,NA,AMP,AML,P,S,OX,KF \\
\hline S66(Escherichia coli) & CIP,VA,NV,CAR,NA,AMP,E,P,S,OX,KF \\
\hline S67, S70 (Staphylococcus sp.) & VA,CAR,NA,AMP,AML,E,K,C,P,S,OX,KF \\
\hline S73, S82 (Streptococcus sp.) & TE,CN,NV,CAR,NA,AMP,P,S,OX,KF \\
\hline S76 (Escherichia coli) & VA,NV,AMP,AML,E,P,OX,KF \\
\hline S78 (Bacillus sp.) & CAR,NA,AMP,AML,E,C,P,OX,KF \\
\hline S83 (Streptococcus sp.) & VA,NV,CAR,NA,AMP,P,S,OX,KF \\
\hline S88 (Enterococcus sp.) & CIP,TE,CN,NV,CAR,NA,AMP,E,K,C,P,S,OX,KF \\
\hline S89, S130 (Escherichia coli) & VA,NV,AMP,AML,E,C,P,OX,KF \\
\hline S93, S96 (Bacillus sp.) & CAR,NA,AMP,C,P \\
\hline S97 (Pseudomonas sp.) & CIP,TE,VA,NV,NA,AML,E,K,C,P,OX,KF \\
\hline S100, S106 (Enterococcus sp.) & CIP,TE,VA,CN,NV,CAR,NA,AMP,E,K,C,P,S,OX,KF \\
\hline S108 (Streptococcus sp.) & CIP,NV,CAR,NA,AMP,P,S,KF \\
\hline S112 (Streptococcus sp.) & CIP,NV,NA,AMP,P,S,KF \\
\hline S114 (Staphylococcus sp.) & VA,CAR,NA,AMP,C,P,S \\
\hline S118 (Streptococcus sp.) & CIP,NV,NA,AMP,C,P,S,KF \\
\hline S125, S128 (Streptococcus sp.) & CIP,NV,NA,C,P,S,OX,KF \\
\hline S132, S137 (Staphylococcus sp.) & VA,NV,CAR,NA,AMP,E,C,P,S, \\
\hline
\end{tabular}




\section{Antibiotic resistance among teeth isolates}

The antibacterial susceptibility test of bacterial isolates is important for choosing appropriate therapy for infections in critically ill patients. Figure (3) showed the clear trend of increasing in the antibiotic resistance ability among the strong biofilm former isolates from teeth $(n=32)$. The high percentages of resistance were $100 \%, 97 \%$ and $94 \%$ for streptomycin, novobiocin and nalidixic acid respectively, followed by kanamycin (75\%), penicillin (75\%), tetracycline $(72 \%)$, chloramphenicol $(69 \%)$, erythromycin $(69 \%)$, oxacillin $(69 \%)$, carbenicillin $(66 \%)$, amoxicillin $(63 \%)$, cephalothin $(59 \%)$, ampicillin $(59 \%)$, gentamicin $(59 \%)$, vancomycin $(59 \%)$ and ciprofloxacin (56\%). Erythromycin has been recommended as an alternative option for patients who are allergic to penicillin and are also widely used for antibiotic prophylaxis of endocarditis associated with dental procedures [18], in our results the isolates have been showed high resistance to erythromycin whereas 22 from 32 isolates were resistant to erythromycin. Another study recommended that amoxicillin and penicillin $\mathrm{G}$ are the most effective antibacterial drugs for the treatment of dental caries [19], in this study the isolates from teeth showed high resistance to both antibiotics recommended by the previously mentioned study. The results of the antibiotic resistance ability of the strong biofilm former isolates from teeth are shown in the table (5). The significant outcome of the results was the high resistance of Enterococcus sp. isolates to all types of antibiotics that used. As mentioned in the literature review, apart from their important role in nosocomial infections, enterococci also play significant roles in dissemination and persistence of antibiotic resistance [20]. The results showed that all the isolates were resistant to the nalidixic acid and this is also documented by another study which mentioned that enterococci possess intrinsic resistance to nalidixic acid [21]. Also, this increasing resistance was observed among the Enterococcus sp. isolates against kanamycin where all the eight isolates were resistant to this antibiotic. Highlevel kanamycin resistance is a well-documented phenomenon among enterococci species [21]. High resistance to novobiocin as observed in the study do not support the results of the previous research of [22]. Table (6) list the antibiogram pattern of the strong biofilm former isolates from teeth.

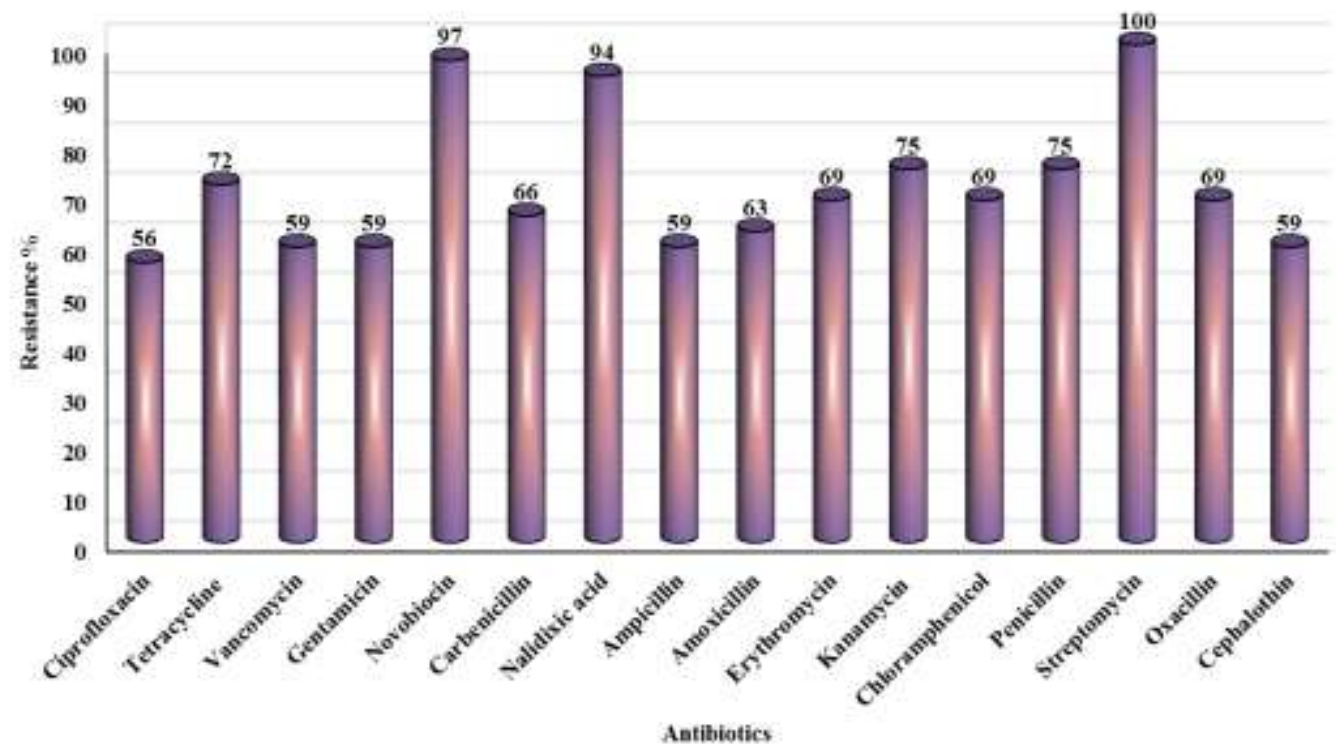

Fig 3 Levels (\%) of antibiotic resistance demonstrated by Strong biofilm producing isolates of Teeth 
Table 5 Numbers of antibiotics resistance isolates from teeth according to genera

\begin{tabular}{|c|c|c|c|c|c|c|c|c|c|}
\hline 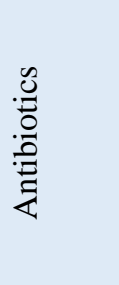 & 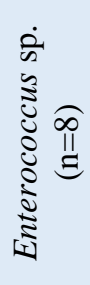 & 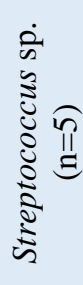 & 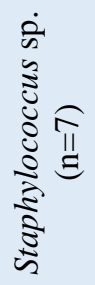 & 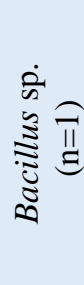 & 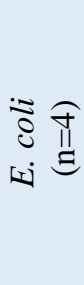 & 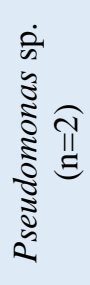 & 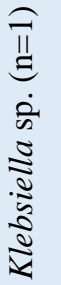 & 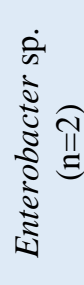 & 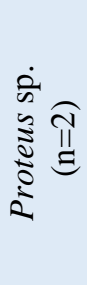 \\
\hline CIP & 8 & 3 & 4 & 0 & 1 & 0 & 0 & 2 & 0 \\
\hline $\mathbf{T}$ & 8 & 4 & 5 & 0 & 1 & 2 & 0 & 1 & 2 \\
\hline $\mathbf{V}$ & 6 & 0 & 2 & 0 & 4 & 2 & 1 & 2 & 2 \\
\hline $\mathbf{C N}$ & 8 & 2 & 5 & 1 & 0 & 2 & 0 & 0 & 1 \\
\hline NV & 8 & 5 & 7 & 0 & 4 & 2 & 1 & 2 & 2 \\
\hline CAR & 6 & 4 & 5 & 0 & 3 & 1 & 0 & 1 & 1 \\
\hline NA & 8 & 5 & 7 & 0 & 3 & 2 & 1 & 2 & 2 \\
\hline AMP & 6 & 1 & 1 & 1 & 3 & 2 & 1 & 2 & 2 \\
\hline AML & 6 & 1 & 3 & 0 & 4 & 2 & 1 & 1 & 2 \\
\hline $\mathbf{E}$ & 8 & 1 & 2 & 0 & 4 & 2 & 1 & 2 & 2 \\
\hline$K$ & 8 & 5 & 7 & 1 & 0 & 2 & 1 & 0 & 0 \\
\hline $\mathbf{C}$ & 8 & 2 & 3 & 1 & 1 & 2 & 1 & 2 & 2 \\
\hline $\mathbf{P}$ & 6 & 3 & 3 & 1 & 4 & 2 & 1 & 2 & 2 \\
\hline $\mathbf{S}$ & 8 & 5 & 7 & 1 & 4 & 2 & 1 & 2 & 2 \\
\hline OX & 6 & 2 & 2 & 1 & 4 & 2 & 1 & 2 & 2 \\
\hline KF & 6 & 5 & 4 & 0 & 1 & 1 & 0 & 1 & 1 \\
\hline
\end{tabular}

Table 6 Antibiogram pattern of the strong biofilm former isolates from teeth

\begin{tabular}{|l|l|}
\hline \multicolumn{1}{|c|}{ Isolates } & \multicolumn{1}{|c|}{ Antibiogram } \\
\hline H1 (Streptococcus sp.) & CIP,TE,NV,CAR,NA,K,S,KF \\
\hline H2 (Staphylococcus sp.) & CIP,TE,CN,NV,CAR,NA,AMP,AML,K,P,S \\
\hline H3 (Bacillus sp.) & CN,AMP,K,C,P,S,OX \\
\hline H4(Escherichia coli) & CIP,VA,NV,CAR,AMP,AML,E,P,S,OX \\
\hline H5, H14, H79, H85 (Enterococcus sp.) & CIP,TE,VA,CN,NV,CAR,NA,AMP,AML,E,K,C,P,S,OX,KF \\
\hline H6(Escherichia coli) & VA,NV,NA,AML,E,P,S,OX \\
\hline H7 (Staphylococcus sp.) & CIP,NV,NA,K,C,S,KF \\
\hline H8 (Pseudomonas sp.) & TE,VA,CN,NV,CAR,NA,AMP,AML,E,K,C,P,SOX,KF \\
\hline H10 (Staphylococcus sp.) & TE,VA,CN,NV,CAR,NA,AML,K,S \\
\hline
\end{tabular}




\begin{tabular}{|c|c|}
\hline H11 (Enterococcus sp.) & CIP,TE,CN,NV,CAR,NA,E,K,C,S \\
\hline H12 (Streptococcus sp.) & CIP,TE,CN,NV,CAR,NA,AMP,AML,K,C,S,KF \\
\hline H19 (Pseudomonas sp.) & TE,VA,CN,NV,NA,AMP,AML,E,K,C,P,SOX \\
\hline H27 (Enterococcus sp.) & CIP,TE,VA,CN,NV,CAR,NA,E,K,C,P,S,OX,KF \\
\hline H30 (Proteus sp.) & TE,VA,NV,CAR,NA,AMP,AML,E,C,P,S,OX \\
\hline H35 (Enterobacter $s p$.) & CIP,VA,NV,NA,AMP,E,C,P,S,OX \\
\hline $\mathbf{H 4 0}$ (Escherichia coli) & TE,VA,NV,CAR,NA,AMP,AML,E,P,S,OX \\
\hline H43 (Proteus sp.) & TE,VA,CN,NV,NA,AMP,AML,E,C,P,S,OX,KF \\
\hline H50 (Streptococcus sp.) & CIP,CN,NV,NA,E,K,P,S,KF \\
\hline H57 (Enterococcus sp.) & CIP,TE,CN,NV,NA,AMP,AML,E,K,C,S \\
\hline H59 (Enterobacter sp.) & CIP,TE,VA,NV,CAR,NA,AMP,AML,E,C,P,S,OX,KF \\
\hline H61 (Staphylococcus sp.) & CIP,NV,CAR,NA,K,P,S \\
\hline H63 (Staphylococcus sp.) & TE,CN,NV,CAR,NA,E,K,C,S,OX \\
\hline H67 (Staphylococcus sp.) & TE,CN,NV,CAR,NA,E,K,C,S,OX,KF \\
\hline H70 (Staphylococcus sp.) & CIP,TE,VA,CN,NV,NA,AML,K,P,S \\
\hline H72 (Enterococcus sp.) & CIP,TE,VA,CN,NV,NA,AMP,AML,E,K,C,P,S,OX,KF \\
\hline H77 (Streptococcus sp.) & TE,NV,CAR,NA,K,C,P,S,OX,KF \\
\hline H80 (Klebsiella sp.) & VA,NV,NA,AMP,AML,E,K,C,P,S,OX \\
\hline H83 (Streptococcus sp.) & TE,NV,CAR,NA,K,P,S,OX,KF \\
\hline H88 (Escherichia coli) & VA,NV,CAR,NA,AMP,AML,E,C,P,S,OX,KF \\
\hline
\end{tabular}

\section{Antibiotic resistance among necrotic roots canals isolates}

The enucleation of infection from the root canal system by means of mechanical and chemical cleansing presents a major hindrance to combat in endodontic treatment. Unfortunately, because of the complex nature of the root canal anatomy, a complete cleansing and disinfection are almost impossible, leaving residual organic and inorganic matter. Pulpal tissue remnants and exudate from the periapical region serve as a substrate for micro-organisms invading the root canal system via either coronal leakage or residing despite treatment [23]. Antibiotic resistance has increased substantially in the recent years and is posing an ever-increasing therapeutic problem [24,25]. It's important to study the ability of the antibiotics resistance of the most prevalence and strong biofilm former isolates present inside the root canal, to present a good understanding of the causes of root canal filling failure or the recurrence of the infection after filling it. After collecting the data of the antibiotic susceptibility test among the strong biofilm former isolates $(n=17)$ from necrotic roots canals, the results showed the increasing ability of these isolates to resist the various 
typed of antibiotics used in this study. The results summarized in figure (4) showed that the percentage of resistance to the kanamycin and streptomycin were $100 \%$, followed by novobiocin $(94 \%)$, ciprofloxacin $(88 \%)$, cephalothin $(88 \%)$, tetracycline $(82 \%)$, gentamicin $(82 \%)$, carbenicillin (82\%), nalidixic acid (82\%), chloramphenicol $(82 \%)$, penicillin $(82 \%)$. Also, the percentages of resistance were $(76 \%)$ for both of ampicillin, amoxicillin and erythromycin, and $(71 \%)$ for oxacillin. The lowest percentage of antibiotic resistance was against vancomycin (53\%). Table (7) shows the number of strong biofilm former isolates resistant to each antibiotic used in this study. For Enterococcus sp., all the isolates $(\mathrm{n}=8)$ resisted to the ciprofloxacin, tetracycline, vancomycin, gentamicin, novobiocin, nalidixic acid, ampicillin, amoxicillin, erythromycin, kanamycin, chloramphenicol, streptomycin. Enterococci have emerged as important nosocomial pathogens and emergence of resistance to many of the antimicrobials used for Gram-positive organisms has made the management of infections due to Enterococcus species difficult [26]. The propensity of Enterococcus species to easily acquire resistance genes and the presence of some unique mechanisms conferring resistance to antibiotics like aminoglycosides and glycopeptide has severely limited the choices available for treating serious infections due to these organisms [27]. The emergence of multi-drug resistant enterococci has led to a scenario which is almost as bad as the preantibiotic era since many of these multi-drug resistant (MDR) strains have developed resistance to practically all available antibiotics [27]. Vancomycin resistance among Enterococcus isolates a major problem in most of the western world, especially in the United States where according to the National Nosocomial Infections Surveillance (NNIS) data, more than 28 percent of all nosocomial enterococcal strains are vancomycin resistant [25]. Table (8) showed the antibiogram of the isolates from necrotic roots canals.

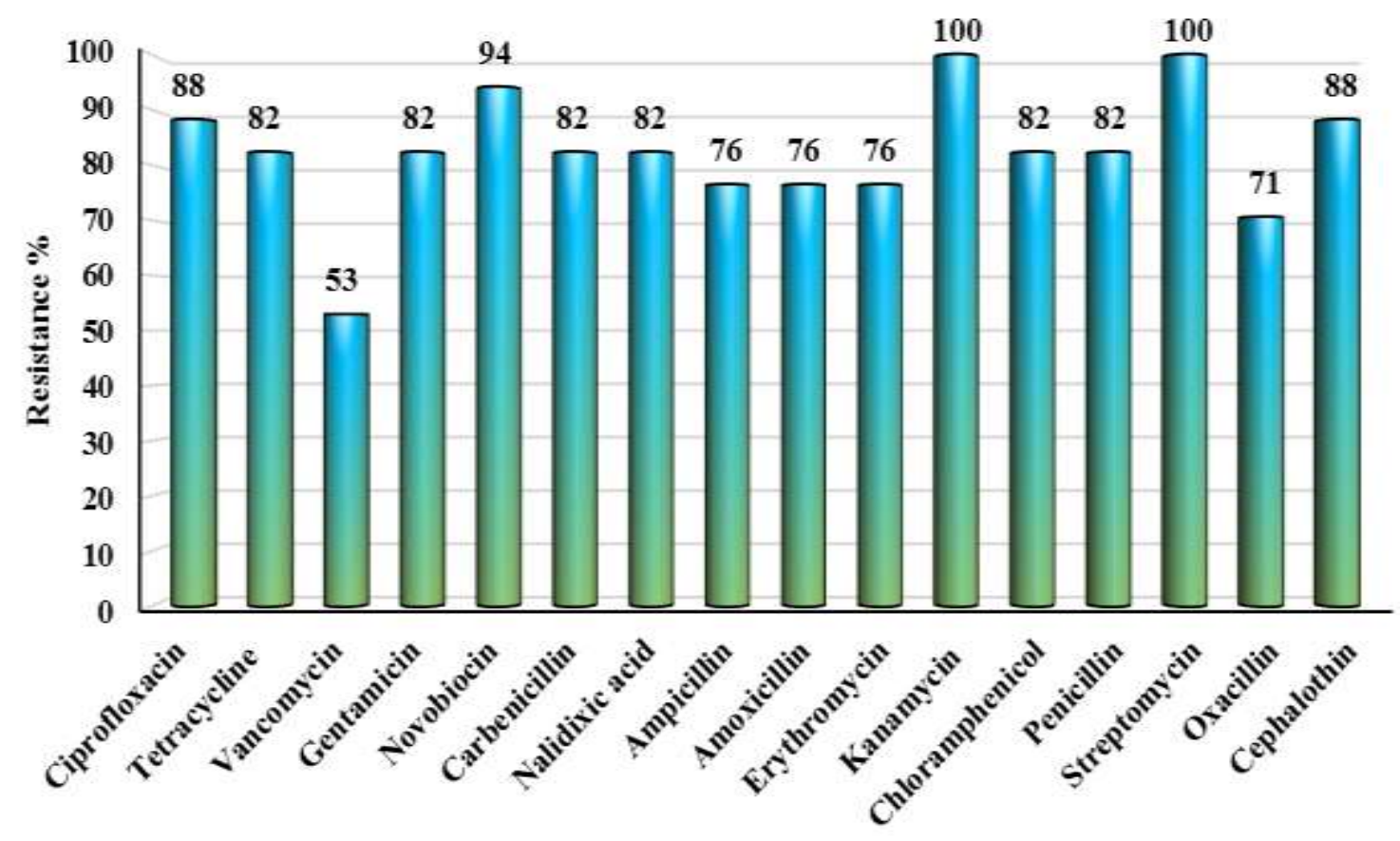

Antibiotics

Fig4 Levels (\%) of antibiotic resistance demonstrated by Strong biofilm producing isolates of necrotic root canals 
Table (7) Numbers of antibiotics resistance isolates from necrotic roots canals according to genera

\begin{tabular}{|c|c|c|c|c|c|}
\hline Antibiotics & $\begin{array}{c}\text { Enterococcus } \\
\mathrm{sp} .(\mathrm{n}=8)\end{array}$ & $\begin{array}{c}\text { Streptococcus } \\
\mathrm{sp} .(\mathrm{n}=5)\end{array}$ & $\begin{array}{c}\text { Staphylococcus } \\
\mathrm{sp} .(\mathrm{n}=1)\end{array}$ & $\begin{array}{c}\text { Bacillus } \\
\mathrm{sp} .(\mathrm{n}=1)\end{array}$ & $\begin{array}{c}\text { Clostridium } \\
\mathrm{sp} .(\mathrm{n}=2)\end{array}$ \\
\hline Ciprofloxacin & 8 & 3 & 1 & 1 & 2 \\
\hline Tetracycline & 8 & 3 & 1 & 0 & 2 \\
\hline Vancomycin & 8 & 0 & 1 & 1 & 0 \\
\hline Gentamicin & 8 & 2 & 1 & 0 & 2 \\
\hline Novobiocin & 8 & 5 & 1 & 1 & 2 \\
\hline Carbenicillin & 6 & 4 & 1 & 0 & 0 \\
\hline Nalidixic acid & 8 & 5 & 0 & 1 & 2 \\
\hline Ampicillin & 8 & 2 & 0 & 1 & 2 \\
\hline Amoxicillin & 8 & 2 & 1 & 1 & 2 \\
\hline Erythromycin & 8 & 1 & 1 & 1 & 2 \\
\hline Kanamycin & 8 & 5 & 1 & 1 & 2 \\
\hline Chloramphenicol & 8 & 2 & 1 & 1 & 2 \\
\hline Penicillin & 7 & 3 & 1 & 1 & 2 \\
\hline Streptomycin & 8 & 5 & 1 & 1 & 2 \\
\hline Oxacillin & 7 & & 1 & 1 & 2 \\
\hline Cephalothin & 7 & 5 & 1 & 1 & 2 \\
\hline
\end{tabular}

Table $8 \quad$ Antibiogram pattern of the strong biofilm former isolates from teeth

\begin{tabular}{|l|l|}
\hline \multicolumn{1}{|c|}{ Isolates codes } & \multicolumn{1}{c|}{ Antibiogram } \\
\hline $\begin{array}{l}\text { M1, M2, M10, M20, M39, } \\
\text { M40 (Enterococcus } \text { sp.) }\end{array}$ & CIP,TE,VA,CN,NV,CAR,NA,AMP,AML,E,K,C,P,S,OX,KF \\
\hline M4, M22 (Clostridium sp.) & CIP,TE,CN,NV,CAR,AMP,AML,E,K,C,P,S,OX,KF \\
\hline M6 (Streptococcus sp.) & CIP,NV,CAR,NA,AMP,AML,K,C,P,S,KF \\
\hline M12 (Streptococcus sp.) & CIP,CN,NV,CAR,NA,K,P,S,KF \\
\hline M18 (Streptococcus sp.) & CIP,TE,CN,NV,CAR,NA,AMP,AML,E,K,P,S,KF \\
\hline M28 (Bacillus sp.) & CIP,CN,CAR,AMP,AML,E,K,C,P,S,OX \\
\hline M29 (Enterococcus sp.) & CIP,TE,VA,CN,NV,NA,AMP,AML,E,K,C,S,OX,KF \\
\hline M31 (Streptococcus sp.) & TE,NV,CAR,NA,K,C,S,OX,KF \\
\hline M34 (Enterococcus sp.) & CIP,TE,VA,CN,NV,NA,AMP,AML,E,K,C,P,S \\
\hline M37 (Streptococcus sp.) & TE,NV,NA,K,S,OX,KF \\
\hline M41 (Staphylococcus sp.) & CIP,TE,VA,CN,NV,CAR,NA,E,K,C,P,S,KF \\
\hline
\end{tabular}

\section{CONCLUSION}

It was concluded that almost all the bacterial isolates in this study can form visible growing biofilms when compared to the controls, though with difference in the degree of adhesions (strong, moderate and weak). The findings revealed that Enterococcus sp. isolated from saliva, teeth and necrotic roots canals was the strongest biofilm former bacteria. Further, the 
study revealed that antimicrobial resistance of bacteria isolated from saliva, teeth and necrotic roots canals of patients with dental caries conditions is associated with biofilm formation.

\section{ACKNOWLEDGMENTS}

The authors are grateful to the School of Biosciences and Biotechnology and the Faculty of Science and Technology, National University Malaysia for providing financial and moral support by grants 04-01-02-SF1244 from Ministry of Science, Technology and Innovation (MOSTI) of Malaysia. The authors also appreciate the stuff of the Dental Clinic, Health Clinic Centre, Seri kembangan, Selangor and Dental Clinic, UKM/Bangi in Malaysia.

\section{REFERENCES}

[1] Seville, L. A., Patterson, A. J., Scott, K. P., Mullany, P., Quail, M. A., Parkhill, J., ... \& Roberts, A. P. 2009. Distribution of tetracycline and erythromycin resistance genes among human oral and fecal metagenomic DNA. Microbial Drug Resistance, 15(3), 159-166.

[2] Patel, R. 2005. Biofilms and antimicrobial resistance. Clinical Orthopaedics and Related Research, 437, 41-47.

[3] Otto, M. 2008. Staphylococcal biofilms. In Bacterial biofilms (pp. 207-228). Springer, Berlin, Heidelberg.

[4] Anderson, G. G., \& O'toole, G. A. 2008. Innate and induced resistance mechanisms of bacterial biofilms. In Bacterial Biofilms (pp. 85-105). Springer Berlin Heidelberg.

[5] Szomolay, B., Klapper, I., Dockery, J., \& Stewart, P. S. (2005). Adaptive responses to antimicrobial agents in biofilms. Environmental Microbiology, 7(8), 1186-1191.

[6] Bagge, N., Schuster, M., Hentzer, M., Ciofu, O., Givskov, M., Greenberg, E. P., \& Høiby, N. 2004. Pseudomonas aeruginosa biofilms exposed to imipenem exhibit changes in global gene expression and $\beta$-lactamase and alginate production. Antimicrobial Agents and Chemotherapy, 48(4), 1175-1187.

[7] Keren, I., Kaldalu, N., Spoering, A., Wang, Y., \& Lewis, K. 2004. Persister cells and tolerance to antimicrobials. FEMS Microbiology Letters, 230(1), 13-18.

[8] Lewis, K. 2005. Persister cells and the riddle of biofilm survival. Biochemistry (Moscow), 70(2), 267-274.

[9] Wood, S. N. 2006. Generalized additive models: an introduction with R. Chapman and Hall/CRC.

[10] Elhadidy, M., \& Elsayyad, A. 2013. Uncommitted role of Enterococcal surface protein, Esp, and origin of isolates on biofilm production by Enterococcus faecalis isolated from bovine mastitis. Journal of Microbiology, Immunology and Infection, 46(2), 8084.

[11] Stepanović, S., Vuković, D., Hola, V., BONAVENTURA, G. D., Djukić, S., Ćirković, I., \& Ruzicka, F. (2007). Quantification of biofilm in microtiter plates: overview of testing conditions and practical recommendations for assessment of biofilm production by staphylococci. Apmis, 115(8), 891-899.

[12] Bauer, A. W., Kirby, W. M., Sherris, J. C., \& Turck, M. 1966. Antibiotic susceptibility testing by a standardized single disk method. American Journal of Clinical Pathology, 45(4), 493.

[13] Peciuliene, V., Balciuniene, I., Eriksen, H. M. \& Haapasalo, M. 2000. Isolation of Enterococcus faecalis in previously root-filled canals in a lithuanian population. Journal of Endodontics, 26(10): 593-595.

[14] Joyanes, P., Pascual, A., Martinez-Martinez, L., Hevia, A. \& Perea, E. 2000. In vitro adherence of Enterococcus faecalis and Enterococcus faecium to urinary catheters. European Journal of Clinical Microbiology and Infectious Diseases, 19(2): 124-127. 
[15] Mohamed, J. A., \& Huang, D. B. 2007. Biofilm formation by enterococci. Journal of Medical Microbiology, 56(12), 1581-1588.

[16] Miller, W. R., Munita, J. M. \& Arias, C. A. 2014. Mechanisms of antibiotic resistance in Enterococci. Expert review of anti-infective therapy, 12(10): 1221-1236.

[17] Slama, T. G. 2008. Gram-Negative Antibiotic Resistance: There Is a Price to Pay. Critical Care 12 (4): S4.

[18] Bagg, J., Macfarlane, T. W., Poxton, I. R. \& Smith, A. 2006. Essentials of microbiology for dental students. Oxford University Press.

[19] Devi, A., Singh, V. \& Bhatt, A. 2011. Antibiotic sensitivity pattern of Streptococcus against commercially available drugs and comparison with extract of Punica granatum. International Journal of Pharma and BioSciences, 2(2): 504-508.

[20] Arvanitidou, M., Katsouyannopoulos, V. \& Tsakris, A. 2001. Antibiotic resistance patterns of Enterococci isolated from coastal bathing waters. Journal of medical microbiology, 50(11): 1001-1005.

[21] Murray, B. E. 1990. The life and times of the Enterococcus. Clinical Microbiology Reviews, 3(1), 46-65.

[22] Manjunath, G., Prakash, R. \& Vamseedhar Annam, K. S. 2011. Changing trends in the spectrum of antimicrobial drug resistance pattern of uropathogens isolated from hospitals and community patients with urinary tract infections in Tumkur and Bangalore. International Journal of Biological and Medical Research. 2(2): 504-507.

[23] Vidana, R., Sullivan, Å. Billström, H., Ahlquist, M. \& Lund, B. 2011. Enterococcus faecalis Infection in Root Canals-Host-Derived or Exogenous Source. Letters in Applied Microbiology 52(2): 109-115.

[24] Austin, D. J., Kristinsson, K. G. \& Anderson, R. M. 1999. The relationship between the volume of antimicrobial consumption in human communities and the frequency of resistance. Proceedings of the National Academy of Sciences, 96(3): 1152-1156.

[25] Guillemot, D. 1999. Antibiotic Use in Humans and Bacterial Resistance. Current Opinion in Microbiology 2(5): 494-498.

[26] Praharaj, I., Sujatha, S. \& Parija, S. C. 2013. Phenotypic \& Genotypic Characterization of Vancomycin Resistant Enterococcus Isolates from Clinical Specimens. The Indian journal of medical research, 138(4): 549.

[27] Arias, C. A. \& Murray, B. E. 2009. Antibiotic-resistant bugs in the 21st century-a clinical super-challenge. New England Journal of Medicine, 360(5): 439-443. 